\title{
Cytology of Extraneural Metastases of Nonhematolymphoid Primary Central Nervous System Tumors: Six Cases with Histopathological Correlation and Literature Update
}

\author{
Joerg Schwock ${ }^{\text {a, b }}$ Lorna Mirham ${ }^{b, c}$ Zeina Ghorab ${ }^{\text {b, d }}$ \\ ${ }^{a}$ Division of Pathology, University Health Network, Toronto, ON, Canada; bepartment of Laboratory Medicine \\ and Pathobiology, University of Toronto, Toronto, ON, Canada; 'Department of Laboratory Medicine, North York \\ General Hospital, Toronto, ON, Canada; 'Division of Anatomic Pathology, Sunnybrook Health Sciences Centre, \\ Toronto, ON, Canada
}

\section{Established Facts}

- Fine-needle aspiration allows for a fast, safe, and accurate diagnosis even in rare conditions such as extraneural metastases of primary central nervous system tumors.

- Depending on latency period, anatomical location of the metastatic manifestation, and primary tumor morphology, cytopathologists will be faced with a potentially complex set of differential diagnoses.

\section{Novel Insights}

- Fine-needle aspiration can be relied upon for the diagnosis of extraneural metastases even in cases with rare morphological and immunophenotypical features such as glioblastoma with a primitive neuronal component if careful correlation with clinical history and primary tumor type is possible.

- Metastases presenting in the head and neck region can mimic a salivary gland pathology, but awareness of the patient's history and appropriately selected ancillary studies can support the cytopathological diagnosis and, consequently, help avoid more invasive sampling modalities.

\section{Keywords}

Fine needle aspiration · Metastasis · Glioblastoma · Salivary gland Extraneural metastases

\section{Abstract \\ Introduction: Extraneural/-cranial metastases (ENM) of pri- mary central nervous system (CNS) tumors are rare and may}

be diagnostically challenging. We describe the cytomorphological and pertinent clinical features of ENM in a case series assessed by fine-needle aspiration (FNA). A search of the laboratory information systems of 2 tertiary care centers in Toronto (2000-2015) was performed. Cases with direct extracranial/-spinal extension of CNS neoplasms were excluded. Microscopic slides of FNA and surgical specimens were reviewed. Demographic and clinicopathological data
(C) 2021 The Author(s)

Published by S. Karger AG, Basel

This is an Open Access article licensed under the Creative Commons Attribution-NonCommercial-4.0 International License (CC BY-NC) (http://www.karger.com/Services/OpenAccessLicense), applicable to the online version of the article only. Usage and distribution for commercial purposes requires written permission.
Correspondence to:

Joerg Schwock, joerg.schwock@uhn.ca 
were retrieved. Case Presentation: Six cases were identified with the original diagnoses of glioblastoma, glioblastoma with primitive neuroectodermal tumor-like components, anaplastic ependymoma, myxopapillary ependymoma, atypical meningioma, and hemangiopericytoma. Median patient age at first diagnosis was 44 years (range 22-56). The time interval between initial diagnosis and first metastatic disease manifestation was 3 months to 19 years. All FNA diagnoses were rendered correctly. In 4 cases, immunohistochemistry was used to support the diagnosis. All cases had prior surgical intervention at the primary tumor site. In 4 cases, the ENM location was the ipsilateral parotid or buccal area. Two primary tumors in midline location developed ENM in the scapular area. Discussion/Conclusion: ENM are a rare manifestation of a range of different primary CNS tumors and may involve the ipsilateral head and neck mimicking clinically a salivary gland neoplasm. FNA can rapidly discriminate ENM from other, potentially more indolent conditions. Awareness of the clinical history is paramount to avoid diagnostic confusion.

(c) 2021 The Author(s)

Published by S. Karger AG, Basel

\section{Introduction}

The incidence of primary central nervous system (CNS) tumors varies by histological type, age at diagnosis, sex, and race/ethnicity. The most common tumor with benign behavior is meningioma, while the most common malignant tumor is glioblastoma, which also carries the poorest prognosis [1]. The projected age-standardized incidence rate for CNS cancer in Canada in 2020 for both sexes is 7.1, and the age-standardized mortality rate is 5.8 [2]. In contrast to brain metastases arising from extracranial/-spinal tumors, which are exceedingly common, extraneural/-cranial metastases (ENM) of primary CNS tumors are rare. However, several cases of different CNS tumor entities presenting with ENM have been reported, mostly as single case reports or small case series. Although data are limited, the incidence of ENM is likely $<1 \%$ for the adult population $[3,4]$. Bone, lymph nodes, and lung are frequently reported anatomical sites involved by metastases from primary CNS neoplasms. Most cases of ENM have been reported in the context of prior surgery or ventriculoperitoneal shunt placement [5]. However, this association with surgical intervention has not been universal $[6,7]$. Different factors have been attributed to the rare occurrence of ENM including (1) physical hindrance of cell migration by the blood-brain barrier and differences in innate defense mechanisms on either side of the barrier, (2) intra-/extraneural dissimilarities in extracellular matrix and adhesion molecule profiles, and (3) the natural course of many brain tumors resulting in patient demise before clinical detectability of the metastases [5].

One large prior series of 16 cases focused on the cytological findings associated with ENM [8]. Fine-needle aspiration (FNA) is frequently the first sampling modality used to examine a newly developed or clinically detected mass. Depending on the anatomical site sampled, cytomorphological assessment may lead to a series of differential diagnoses. These are used to either generate a final diagnosis if the morphological findings and clinical context permit or require further analysis with carefully selected ancillary studies. Because of its rarity, ENM may not be included in the differential diagnosis (DDx) if the clinical history of the patient is not provided or ignored. Lack of appropriate clinical history may be more likely encountered with an increasing latency from the initial diagnosis of the CNS neoplasm or if the clinical presentation is compelling enough to favor a specific alternative diagnosis (e.g., an inflammatory process). In such circumstances, the FNA sample assessment can be particularly challenging and may result in a misdiagnosis and/or otherwise avoidable and increasingly invasive repeat sampling procedures. Compounding this difficulty is the fact that cytomorphology by itself can overlap with other, more frequent entities and/or appear bland in some instances belying the poor long-term prognosis imparted by ENM in general.

We examined the cytomorphological findings of a case series of ENM from 2 tertiary care hospitals with predominantly adult patient population and correlated them with histopathological features. Due to a recurrent involvement of the parotid gland area in our series, we specifically reviewed the literature for similar cases in order to provide some guidance for clinical and cytopathological assessment in this prognostically poor patient population.

\section{Materials and Methods}

Natural language searches of the laboratory information systems of 2 tertiary care centers (University Health Network, Sunnybrook Health Sciences Centre) were performed including the years 2000-2015 and using the following terms: glioblastoma, pericytoma, hemangiopericytoma, glioma, ependymoma, choroid plexus, meningioma, germinoma, medulloblastoma, gliomatosis, pineoblastoma, astrocytoma, and oligodendroglioma. The electronic patient records of patients with suspected ENM were reviewed, and relevant available microscopic slides of confirmed 


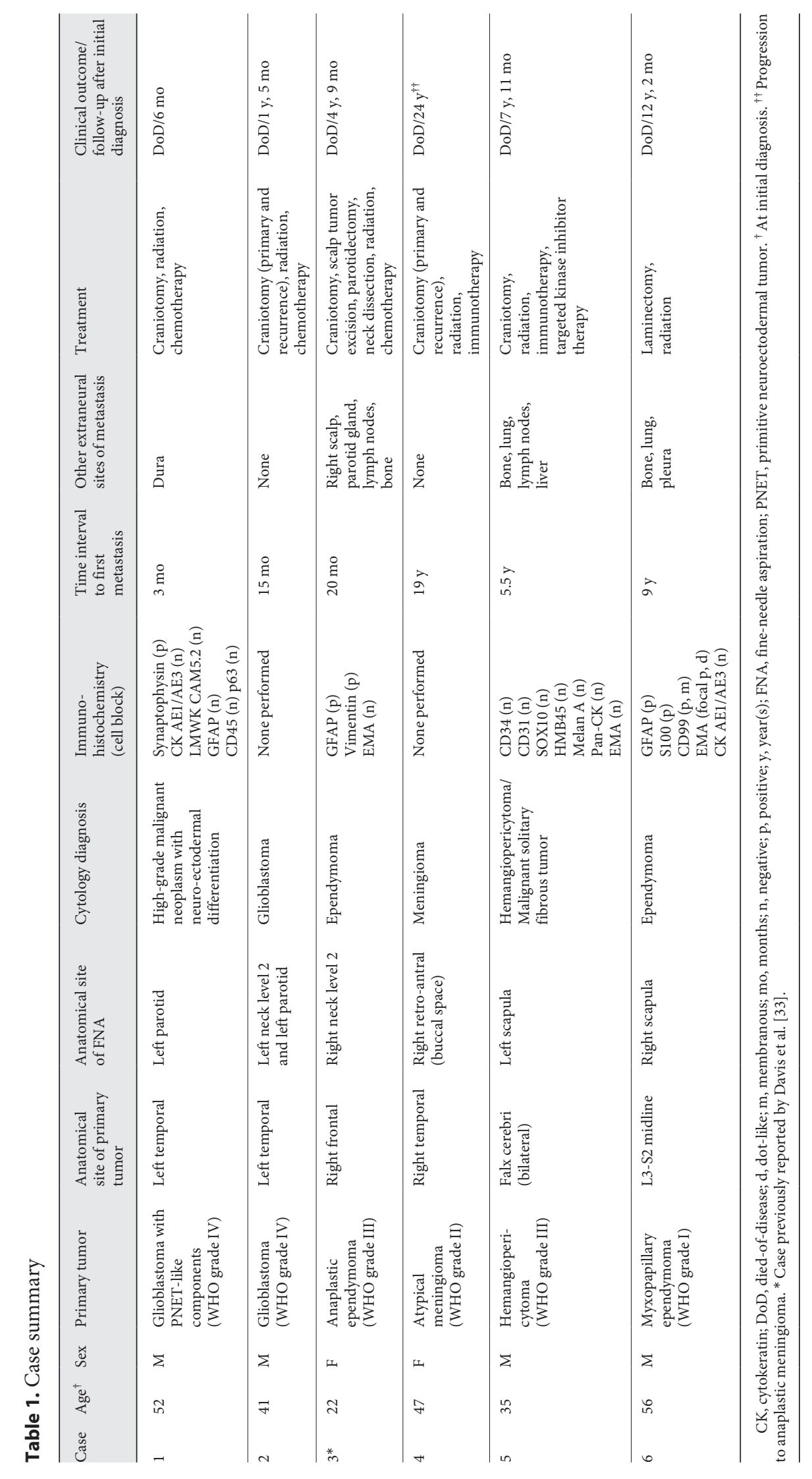


Fig. 1. Glioblastoma with a primitive neuronal component. a Dyshesive malignant cells of relatively small size contain nuclei with granular chromatin and inconspicuous nucleoli, and display synaptophysin reactivity (inset) in a left parotid gland FNA (Papanicolaou-stained cytocentrifuge slide $\times 63$ oil; inset: immunohistochemistry $\times 40$ ). b Identical morphological features including synaptophysin reactivity (inset) are present in the prior surgical resection of the left temporal brain tumor (HE-stained tissue section. $\times 63$ oil; inset: immunohistochemistry $\times 40)$. FNA, fine-needle aspiration.

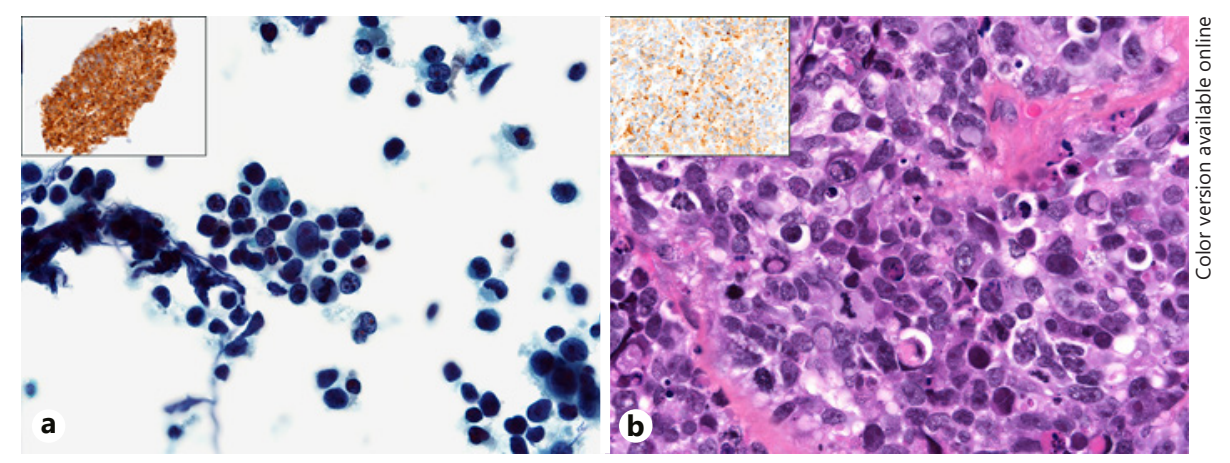

cases were retrieved for review. FNA was performed with 22-25 G needles (where information available) under image guidance by clinicians and radiologists. The specimens were prepared on-site into direct smears or submitted in appropriate cytology fixatives (CytoLyt ${ }^{\circledR}$, Saccomanno) for liquid-based cytology (ThinPrep ${ }^{\circledR}$ ) or cytocentrifuge preparations produced in the institutional laboratories. Papanicolaou or Field's stain was used as appropriate for alcohol-fixed and air-dried slides, respectively. Cellblocks were prepared either with HistoGel ${ }^{\mathrm{TM}}$ or using the plasma-thrombin method followed by formalin postfixation. In cases with on-site assessment, balanced saline solution needle rinse fluid was used as substrate for cellblocks. Immunohistochemical studies were performed on formalin-fixed, paraffin-embedded materials using standard methodologies. Appropriate controls were included. To specifically examine the extant literature with respect to parotid gland involvement by metastatic glioblastoma, a PubMed ${ }^{\circledR}$ search was conducted (www.pubmed.ncbi.nlm.nih.gov/) with "parotid" AND "glioblastoma" as terms (date: January 18, 2021). Reports of ENM arising from other primary CNS tumors, those that reported involvement of neck lymph nodes without stating the parotid gland, and those unrelated to the subject area were removed. Thirteen publications were included, and an additional case report was identified by review of the references.

\section{Results}

\section{Patient Population}

Six cases with single site or disseminated ENM of primary CNS tumors were identified. The primary diagnoses were glioblastoma $(n=2$, including one case of glioblastoma with primitive neuroectodermal tumor [PNET]like components), ependymoma ( $n=2$, one case each of anaplastic and myxopapillary type), atypical meningioma $(n=1)$, and hemangiopericytoma $(n=1$, now termed solitary fibrous tumor) (Table 1). Median patient age at first diagnosis was 44 years (range 22-56). The interval between initial diagnosis and ENM varied widely (3 months to 19 years). All patients succumbed to the dis- ease. All cases had a previous surgical intervention at the primary site. Four cases with primary tumors located to the right or left of the midline presented with ipsilateral ENM in the area of the parotid gland, cheek soft tissue, and neck, which raised the DDx of a synchronous or metachronous primary neoplasm of head and neck origin such as a salivary gland tumor. Two patients with midline tumors (cases 5 and 6) had metastases to the left and right scapula, respectively, and eventually developed widespread metastatic disease. ENM including tumor type was correctly diagnosed by FNA in all cases. The FNA diagnosis was supported by immunohistochemical studies in 4 cases.

\section{Case 1}

A 53-year-old male presented with a left parotid lesion for which FNA was performed. Three months prior, the patient was diagnosed with a left temporal glioblastoma with PNET-like components, WHO grade IV, in a resection performed at a different institution. The FNA specimen revealed poorly differentiated malignant cells disposed singly and in variably sized clusters (Fig. 1a). The cell population consisted predominantly of small cells admixed with intermediate and occasional larger forms. The cells displayed scant cytoplasm and round-ovoid nuclei with granular chromatin. Nucleoli were inconspicuous. Mitotic activity and apoptotic bodies were present. Immunohistochemistry showed positivity for synaptophysin, while stains for cytokeratins (AE1/AE3 and CAM5.2), GFAP, p63, and CD45 were negative (Table 1). On the basis of the morphological and immunophenotypical features, and taking into account the clinical history, the diagnosis of a high-grade malignant neoplasm with neuroectodermal differentiation was rendered and an extracranial metastasis of the previously diagnosed glial neoplasm was favored. Subsequent review of the sections produced 
Fig. 2. Glioblastoma. a Pleomorphic tumor cells with indistinct cytoplasm and prominent, fibrillary processes in a left neck level 2 FNA (Field-stained direct smear $\times 10$ ). b The features are recapitulated in the $\mathrm{CNB}$ obtained from the same site with tumor necrosis (asterisk) and skeletal muscle fibers (right margin) seen in the same field (HEstained tissue section $\times 20$ ). FNA, fine-needle aspiration; $\mathrm{CNB}$, core-needle biopsy.
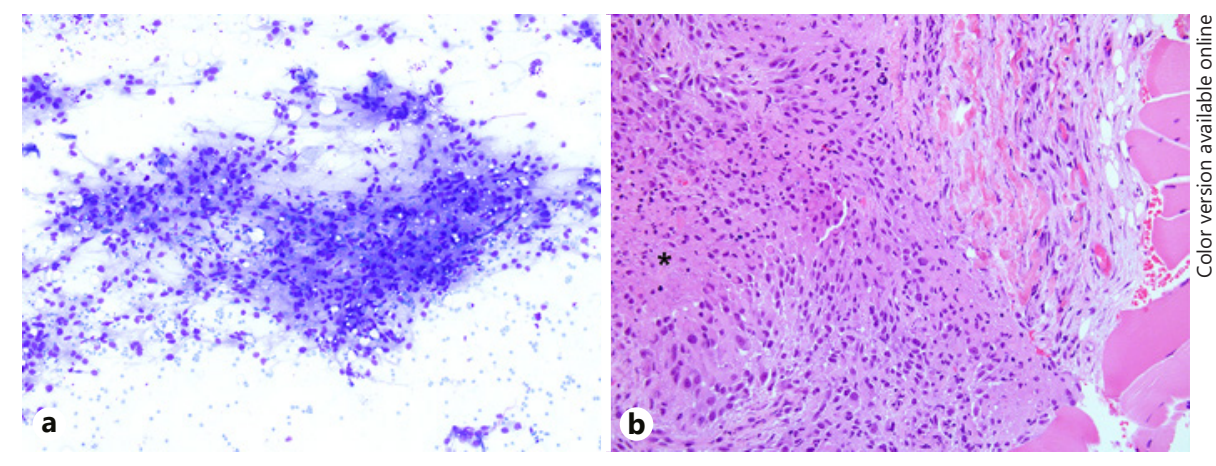

Fig. 3. Anaplastic ependymoma. a Spindle and epithelioid cells in a right neck level 2 FNA with perivascular pseudorosettes (inset) identifiable in the cellblock section ( $\mathrm{Pa}$ panicolaou-stained ThinPrep ${ }^{\circledR}$ slide $\times 63$; inset: HE-stained cellblock section $\times 40$ ). b Metastatic involvement of the parotid gland with pseudorosette arrangement of the tumor cells, focal gemistocytic-like tumor change (asterisk), and adjacent salivary gland parenchyma (HE-stained tissue section $\times 10)$. FNA, fine-needle aspiration.
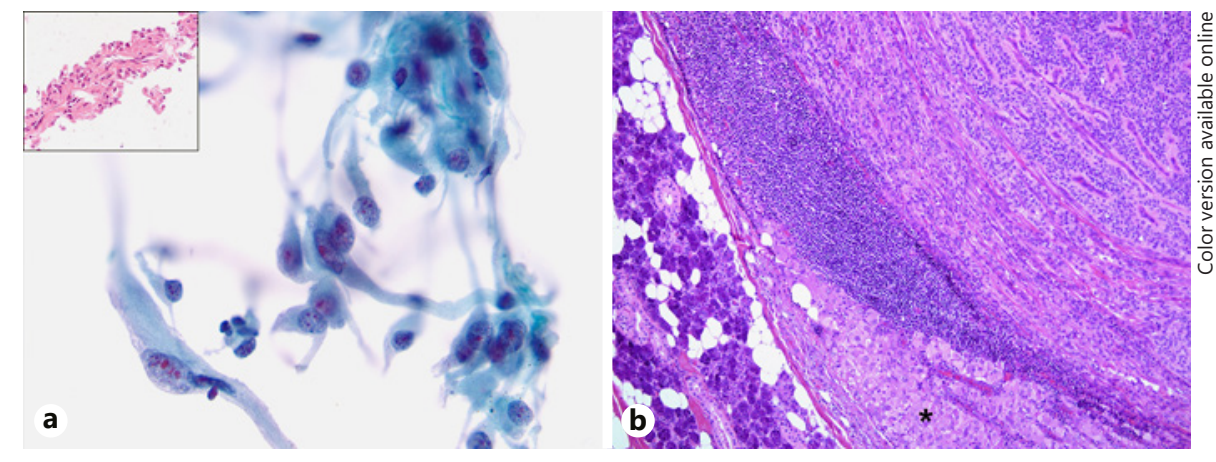

from the brain tumor showed an infiltrating neoplasm made up of 2 distinct, intermingled components. One component displayed astroglial morphology, demonstrated immunohistochemical GFAP expression, and a Ki67 index of approximately $25 \%$. The second tumor component showed "small round blue cell" features, as previously identified in the FNA sample, with diffuse synaptophysin reactivity (Fig. 1b) and a Ki67 index close to $100 \%$ (not shown). Mitotic activity and apoptotic bodies were readily seen in this tumor portion. Diffuse p53 reactivity was noted. Isocitrate dehydrogenase (IDH) R132H immunohistochemistry was negative.

\section{Case 2}

A 42-year-old male presented with a left parotid and a left neck level 2 mass of approximately $2 \mathrm{~cm}$ in each location. Fifteen months prior to that, he had undergone surgery for an IDH R132H-negative left temporal glioblastoma followed by additional debulking surgeries at 6 and 13 months for tumor recurrences. Both the parotid and the level 2 mass were sampled by FNA with on-site evaluation followed by a concurrent core-needle biopsy (CNB) of the level 2 lesion. Microscopic examination of the cytology samples demonstrated poorly circumscribed fragments of neoplastic tissue composed of loosely cohesive, pleomorphic, polygonal, and spindle-shaped cells with fibrillary processes (Fig. 2a) as well as necrotic debris. Proliferating vessels were not conspicuous perhaps because tissue fragments tend to be smaller in FNA samples than intraoperative squash cytology. FNA and CNB (Fig. 2b) both confirmed metastatic involvement by the previously diagnosed glioblastoma. Morphological comparison with a prior debulking specimen (not shown) revealed essentially identical features.

\section{Case 3}

A 25 -year-old female presented with a $1.5-2 \mathrm{~cm}$ mass deep to the sternocleidomastoid muscle in right level $2 \mathrm{~B}$ location. The medical history of the patient was significant for an anaplastic ependymoma involving the right frontal lobe 34 months prior, which was treated with resection and adjuvant radiation. Subsequently, the patient had developed soft tissue metastases including the right postauricular scalp and an enhancing nodule in the right parotid gland seen on computed tomography. The soft tissue metastases had been resected at 20 and 22 months after the initial diagnosis and further radiation treatment were administered. The FNA performed on the right level $2 \mathrm{~B}$ mass displayed a mixture of spindle and epithelioid cells including bizarre, anaplastic forms in a relatively 
Fig. 4. Atypical meningioma. a Relatively monomorphous, loosely cohesive epithelioid cells displaying concentric whorling cell arrangement (arrow) and intranuclear cytoplasmic pseudoinclusions (inset) in a right retro-antral FNA (Field-stained direct smear $\times 40$; inset: $\times 63$ oil). b A prior resection of the recurrent meningioma shows tumor cells in a sheet-like growth pattern with several whorls (arrows) and mitotic activity (inset) (HE-stained tissue section $\times 63$ oil; inset: $\times 63$ oil). FNA, fine-needle aspiration.
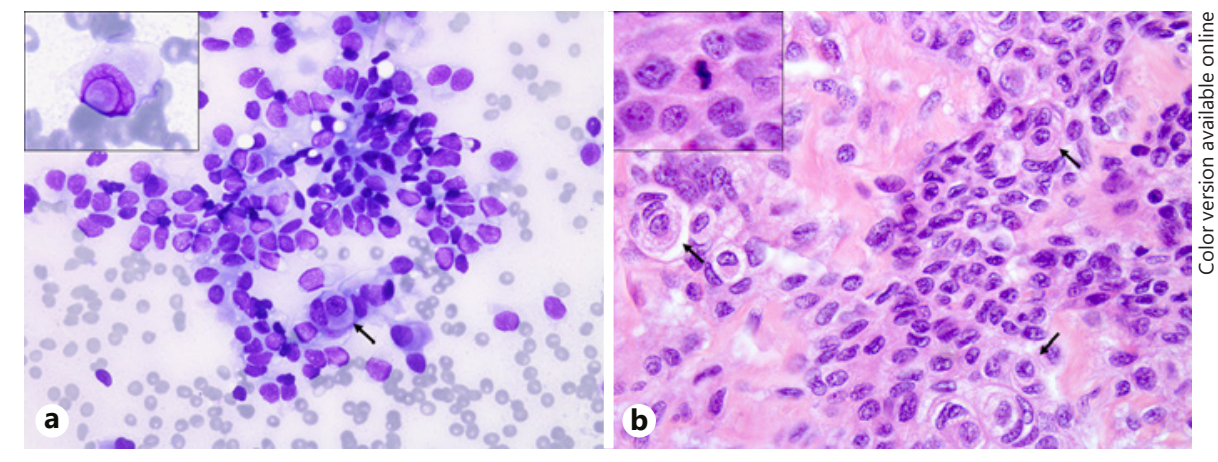

Fig. 5. Solitary fibrous tumor/hemangiopericytoma. a Cohesive tissue fragment comprised of relatively monomorphous, closely apposed cells with small nucleoli (inset) arranged in a haphazard pattern around a thin-walled vessel (asterisk) in a left scapular FNA (HE-stained cellblock section $\times 40$; inset: Papanicolaou-stained cytospin preparation $\times 63$ oil). $\mathbf{b}$ The prior resection demonstrates essentially the same features including multiple mitotic figures (arrows) (HE-stained tissue section $\times 20$ ). FNA, fineneedle aspiration.
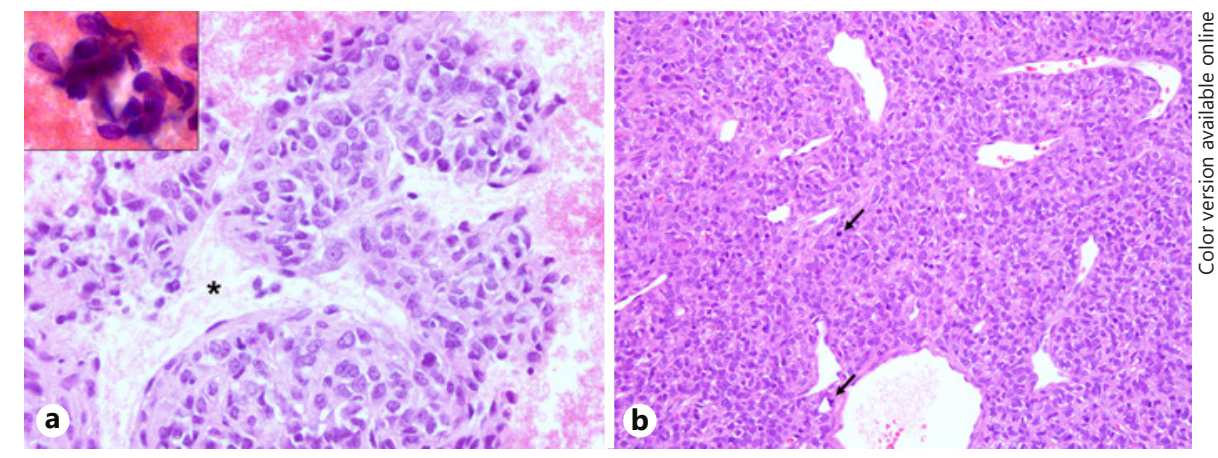

clean background (Fig. 3a). Perivascular pseudorosettes were noted in the cellblock preparation. Immunohistochemistry performed with cellblock sections supported the diagnosis of metastatic ependymoma (Table 1). A right parotidectomy and neck dissection performed 5 months after the FNA revealed metastatic disease involving the gland as well as intra- and extraparotid lymph nodes (Fig. 3b). Intra- and extracranial progression was noted soon after surgery.

\section{Case 4}

A 66-year-old female presented with a new retro-antral (buccal space) $1.5-\mathrm{cm}$ soft tissue nodule in the context of recent treatment for recurrent atypical meningioma. The initial diagnosis of atypical meningioma was rendered 19 years prior after surgical resection of a right temporal tumor with marked cellularity, tumor cell arrangement in sheets, and prominent necrosis. Since this initial surgery, the patient had suffered a recurrence involving the ipsilateral orbit at 8 years, which was treated with radiotherapy and, after further progression at 14 years, with a surgical revision procedure resulting in subtotal resection of the intraorbital tumor component followed by reirradiation. The FNA specimen obtained from the retroantral site showed relatively monomorphous, loosely co- hesive, epithelioid cells with focal concentric whorling (Fig. 4a). The nuclei were round-ovoid. Nucleoli were inconspicuous, and occasional intranuclear cytoplasmic pseudoinclusions were present. The sample had insufficient cellularity for a cellblock preparation. However, comparison with a prior surgical specimen revealed similar morphological features (Fig. 4b). The metastatic tumor was treated with radiation; however, new dural disease was subsequently noted in addition to progressive intraorbital disease. As part of a clinical trial, immunotherapy with nivolumab was administered. A third craniotomy was necessary at 22 years due to the mass effect caused by progressive disease. At this point, the recurrent meningioma displayed anaplastic features. Eventually, multifocal recurrences involving the right orbit and hemisphere developed.

\section{Case 5}

A 41-year-old male had an FNA performed for a lesion located at the left scapular spine. He had a history of solitary fibrous tumor/hemangiopericytoma (WHO grade III), which originally presented with bilateral involvement of the falx cerebri and was treated with surgical resection followed by radiation 5.5 years earlier. The FNA demonstrated the features of a spindle cell neoplasm with 
Fig. 6. Myxopapillary ependymoma. a Stellate neoplastic cells with long cytoplasmic processes and round to ovoid, mildly irregular nuclei organized in perivascular pseudorosettes with GFAP immunoreactivity (inset) in a right scapular FNA (Papanicolaou-stained direct smear $\times 40$; inset: immunohistochemistry $\times 40$ ). b The concurrent core biopsy displays identical features with radially arranged, cuboidal to elongated cells, and central fibrovascular cores (HEstained tissue section $\times 10$ ). FNA, fine-needle aspiration.

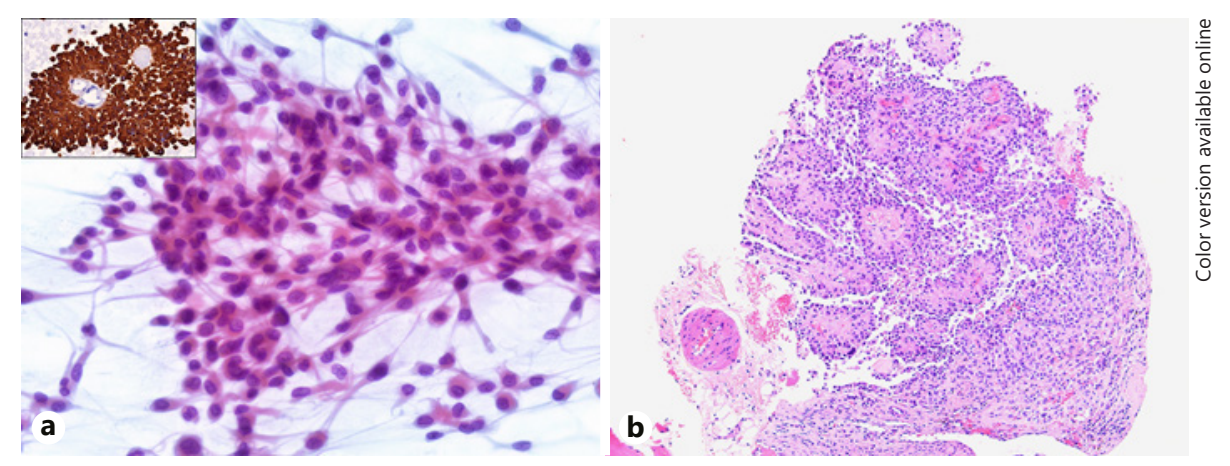

vague storiform-fascicular architecture (Fig. 5a). Most of the tissue was represented in the cellblock section in form of cohesive fragments, which encompassed ectatic, branching blood vessels. The cells displayed scant, eosinophilic cytoplasm, and relatively monomorphous ovoid nuclei with pinpoint nucleoli. Mitoses were inconspicuous in the limited sample. The background contained abundant blood. Immunohistochemistry on the cellblock sections produced negative results for the selected markers with CD31 and CD34 reactivity present only in endothelial cells (Table 1). However, morphological comparison with the original tumor demonstrated essentially identical features supporting the diagnosis of metastatic solitary fibrous tumor/hemangiopericytoma (Fig. 5b). Scattered osteoclast-like giant cells, microcystic change, collagenous areas, focal necrosis, and mitotic activity (8 mitoses/10 high-power fields [field diameter: $0.55 \mathrm{~mm}$ ]) were noted in the original resection but not readily seen in the FNA likely due to the limited quantity of tissue. CD34 immunoreactivity was focal and weak in the original resection, and stains for S100, GFAP, cytokeratin (AE1/AE3), melanoma antibody cocktail, and EMA were negative. Subsequently, disseminated metastatic disease involving the bone, lung, lymph nodes, and liver was found. Treatment consisted of palliative radiation as well as 2 different kinase inhibitors and immunotherapy agents in the context of clinical trials.

\section{Case 6}

A 65-year-old male presented with a lytic lesion of the right scapula for which computed tomography-guided FNA with on-site assessment and concurrent CNB was performed. The patient's history included a prior diagnosis of lumbosacral myxopapillary ependymoma approximately 10 years prior with 2 surgical resections performed 2 months apart followed by adjuvant radiation. A local recurrence was seen 6 years later. The FNA specimen contained round, epithelioid, and stellate neoplastic cells with prominent cytoplasmic processes and fairly well-defined cell borders (Fig. 6a). The round to ovoid, mildly irregular nuclei contained granular chromatin. The slide background contained blood rather than myxoid material. The cellblock section revealed perivascular pseudorosettes with positivity for GFAP and S100, membranous CD99, and focal, dot-like cytoplasmic EMA reactivity supporting the diagnosis of metastatic myxopapillary ependymoma (Table 1). Immunoreactivity for cytokeratin (AE1/AE3) was lacking in both the cellblock and the independently reported CNB (Fig. 6b). The patient was found to have additional lesions involving the bone, lung, and pleura. The subsequent treatment included palliative radiation and surgery for metastatic cord compression.

\section{Discussion}

ENM of primary CNS neoplasms are rare and may cause diagnostic confusion due to potential clinical, cytomorphological, and immunophenotypical overlap with other entities, which enter the DDx depending on the anatomical site of manifestation. Specifically, ENM involving the head and neck region may mimic abnormalities of the salivary glands such as parotitis (esp. if associated with pyrexia, with rapid clinical development and in the immediate postoperative period) [9-11], a primary salivary gland neoplasm $[7,12]$, or other neoplastic processes [13] causing potential for delay in clinical management as well as unnecessary medical interventions. Lymph nodes incorporated into the parotid gland as a result of the gland's embryological development, drain forehead, nasal root, upper lip, cheek, temple, and external ear. As such, most metastases arise from squamous cell carcinomas or melanomas within this region [7]. More uncommonly, distant metastases (breast, lung, kidney, and oth- 
er) manifest themselves in and around the parotid gland. Of note, rare neuroectodermal neoplasms [14] and meningiomas [15] do occur in primary extraneural/-cranial locations. Extracranial meningiomas may present in a variety of sites within the head and neck region (e.g., sinonasal tract, ear and temporal bone, and scalp) $[15,16]$. Distinction between the sites of origin is clinically important since, in contrast to the more common primary intracranial counterparts with extracranial extension or metastasis, the prognosis of the primary extracranial neoplasms is good, in particular if disease recurrence after surgery can be avoided $[14,15]$.

Glioblastoma is the most common malignant brain tumor in adults accounting for approximately $45-50 \%$ of all primary malignant tumors. $I D H$-wild-type glioblastoma is the most common form with about $90 \%$ of all cases and presents at a mean patient age at diagnosis of 62 years in contrast to $\mathrm{IDH}$-mutant glioblastomas, which account for the remaining cases, develop through progression from a lower grade glial tumor, and manifest at a mean age of 45 years. Differentiation of $I D H$-mutant from $I D H$-wildtype glioblastomas is prognostically important. Glioblastoma with PNET-like components (now "glioblastoma with a primitive neuronal component" [17]) is included as subtype of glioblastoma in the 2016 WHO Classification of Tumours of the Central Nervous System [18]. Perry et al. [19] estimated the frequency of PNET-like components in glioblastoma at $0.5 \%$. Glioblastoma with a primitive neuronal component is characterized by an otherwise histologically classic glioblastoma with one or more nodules of primitive neuronal differentiation characterized by markedly increased cellularity, cells with high nucleocytoplasmic ratio, and high mitosis-karyorrhexis index. Homer-Wright rosettes, cell wrapping, and anaplastic cytology may occur. Immunoreactivity for synaptophysin, markedly elevated Ki67, and reduction/ loss of GFAP are useful ancillary features. A high rate (30-40\%) of cerebrospinal fluid dissemination and frequency $(\sim 40 \%)$ of $\mathrm{v}-\mathrm{Myc}$ avian myelocytomatosis viral oncogene neuroblastoma-derived homolog $(M Y C N)$ or MYC amplification are considered distinctive [18, 19]. The presence of mutations in $I D H 1$ or $I D H 2$, strong and extensive p53 immunoreactivity, as seen in case 1 of our series, and clinical history in a subset of cases support that some tumors arise as secondary glioblastomas $[18,20]$. Specifically, Perry et al. [19] reported foci resembling diffuse low-grade glioma in $89 \%$ of their specimens. Relative restriction of the $M Y C$ gene amplification to the primitive neuronal component suggests that it occurs as secondary alteration. Neuroblastic/neuronal metaplasia and clonal expansion of a tumor stem cell/progenitor cell population are 2 hypothetical mechanisms that might explain the occurrence of the subtype [19]. Of note, glioblastoma with a primitive neuronal component should not be confused with small-cell glioblastoma, which features monomorphic, deceptively bland nuclei, and other morphological characteristics that overlap more with oligodendroglial tumors.

ENM arising from glioblastoma with a primitive neuronal component has been reported recently [21, 22]. However, case 1 of our series is, to the best of our knowledge, the first reported case with manifestation in the parotid gland. Our review of the literature resulted in 14 prior cases of reported glioblastoma with parotid gland involvement (Table 2) [6,9-12, 23-31]. Including the 2 cases of our series, patients with parotid gland involvement by glioblastoma presented with a male-to-female ratio of 2.2:1 and a median age of 44 years (range 24-72 years). With the exception of the earliest case reported in 1974 [6], all cases had undergone surgery prior to discovery of the metastases. The laterality of the primary tumors was more commonly left $(10 / 16 ; 62.5 \%)$, and in $14 / 15$ (93\%) cases with reported laterality of the metastasis, the tumor deposit was detected ipsilateral to the primary tumor. FNA was used as first sampling modality of the extraneural lesion in 6/14 (43\%) cases with available information. Interestingly, 3 cases presented with pyrexia, suspected parotitis, and reported initial antibiotic treatment, respectively, triggered by the clinically detected abnormality. In one case, the histomorphological DDx of mucoepidermoid carcinoma was raised.

As demonstrated by cases 3 and 4 of our series, metastases in and around the parotid gland area are not limited to glioblastomas and may also occur with primary CNS neoplasm of lower WHO grade. Indeed, one case of oligodendroglioma, a WHO grade II tumor, with metastasis to the parotid gland after intracranial recurrence and progression to WHO grade III has previously been reported $[8,32]$. Case 3 of our series was previously reported [33]; however, the findings of the FNA were not included. Cytomorphology in conjunction with ancillary studies performed on cellblock sections permitted in this case the accurate diagnosis of metastatic ependymoma. More cases of ENM arising from primary intracranial anaplastic ependymomas have been reported recently [34-37] including one case each with involvement of the mastoid region [36] and the bilateral parotid glands [37]. Classic and anaplastic ependymoma correspond to WHO grades II and III, respectively. However, grading is subjective, no definite association has been established between grade 
Table 2. Summary of glioblastoma cases with parotid gland involvement reported to date

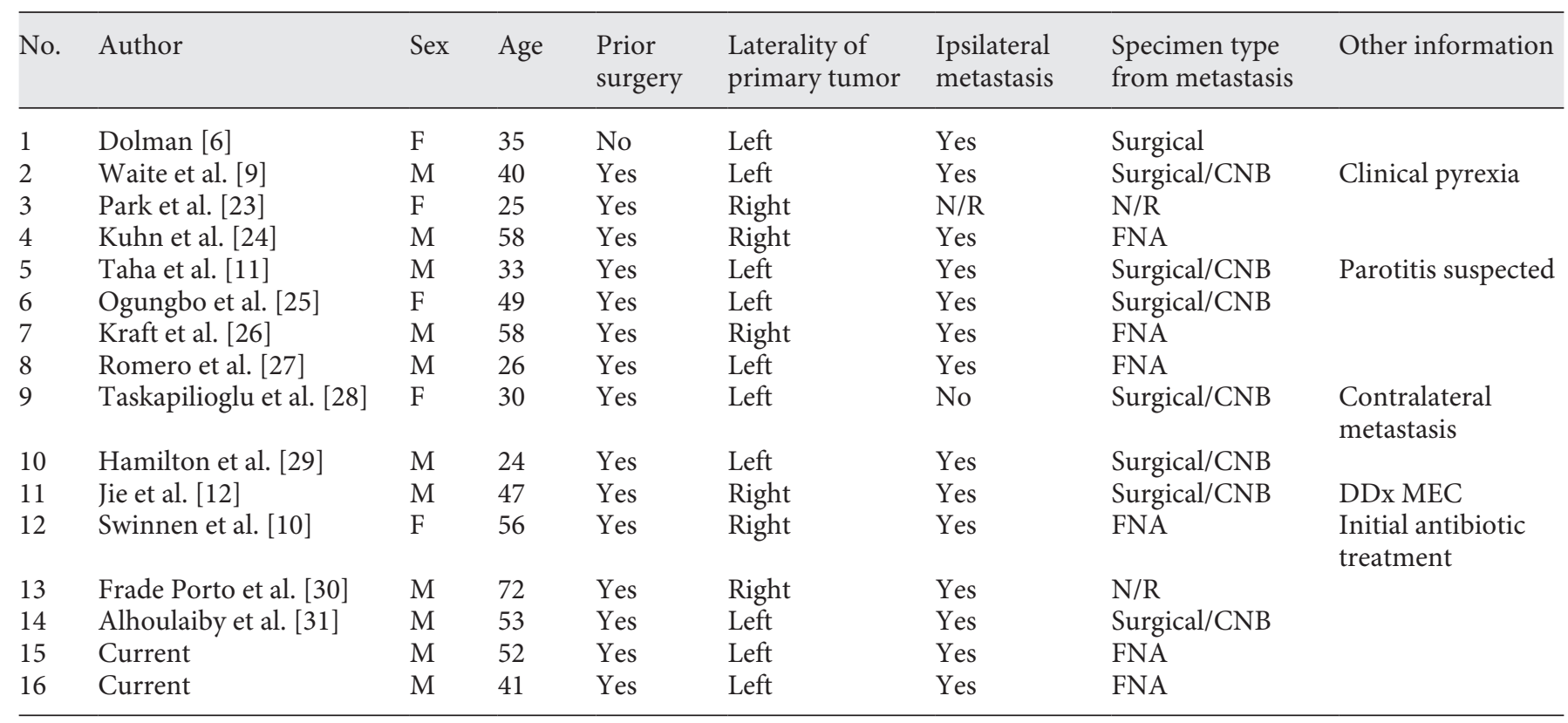

CNB, core-needle biopsy; DDx, differential diagnosis; FNA, fine-needle aspiration; MEC, mucoepidermoid carcinoma; N/R, not reported.

and biological behavior, and grade is not generally used for therapeutic stratification [18]. Ependymomas arise, in order of decreasing frequency, in the posterior fossa, supratentorial, and the spinal canal. Rare primary extraCNS ependymomas do occur [14]. Posterior fossa ependymomas are most common among children, while supratentorial ependymomas are found in both pediatric and adult patients. Spinal tumors such as myxopapillary ependymoma typically occur in young adults. More recently, 9 molecular groups of ependymoma have been described which are associated with differences in anatomical location, patient age, histopathology, and clinical outcome. Supratentorial ependymomas with RELA fusion and posterior fossa ependymomas group A with balanced genome, together mainly seen in the pediatric population, are associated with poor prognosis [18]. The histopathological diagnosis of anaplastic ependymoma depends on "a high cell density and elevated mitotic count alongside widespread microvascular proliferation and necrosis" found in an ependymal tumor [18]. The immunoprofile is no different from classic ependymoma except for a higher proliferative index, yet no Ki67 labeling cutoff is established. Specifically in the context of myxopapillary ependymoma, which manifests in the region of conus medullaris, cauda equina, and filum terminale, pedi- atric patients are prone to spinal metastatic dissemination. Lack of adjuvant therapy and incomplete excision has also been associated with distant failure.

Meningioma is the most frequent intracranial tumor. The vast majority of meningiomas behave in a benign fashion, and females are at greater risk [1]. WHO grade II meningiomas encompass the chordoid, clear cell, and atypical variants, whereas the papillary, rhabdoid, and anaplastic variants are assigned a grade III. Extracranial metastases are rare, occur in about 1:1,000 cases and mostly in the context of grade III tumors [18]. A number of mutations, cytogenetic changes, and gene expression alterations have been detected in meningiomas. NF2 mutations are common in both syndromic and sporadic meningiomas and represent an early tumorigenic event, while alterations of the cyclin-dependent kinase inhibitor $2 \mathrm{~A}(C D K N 2 A)$ gene and the telomerase reverse transcriptase (TERT) gene promoter are associated with a higher WHO grade [18]. Case 4 of our series initially presented as atypical meningioma, which is an entity characterized by an increased mitotic activity ( $\geq 4$ mitoses/10 highpower fields of $0.16 \mathrm{~mm}^{2}$ ), histological brain invasion, or at least 3 of the following features: increased cellularity, small cells with increased nucleocytoplasmic ratio, prominent nucleoli, uninterrupted patternless or sheet-like 
growth, and spontaneous (i.e., noniatrogenic) necrosis [18]. The initial diagnostic report of our case described a markedly cellular tumor with cell arrangement in sheets and prominent necrosis. The mitotic count was $3 / 10$ high-power fields (field area not specified). In contrast, for the diagnosis of anaplastic meningioma overtly malignant cytological features and/or markedly elevated mitotic activity are required [18]. In our patient, resection of a recurrence 6 years prior to death resulted in a diagnosis of recurrent meningioma with anaplastic features due to a mitotic activity of up to 27 mitoses per 10 high-power fields.

Finally, one of our 2 primary intraneural tumors with initial midline location and presentation with ENM to the scapula was a solitary fibrous tumor/hemangiopericytoma. The combined term was introduced in the 2016 WHO classification due to the finding that both the solitary fibrous tumor (characterized by "patternless" architecture, relatively low cellularity and thick bands of collagen) and the hemangiopericytoma phenotype (characterized by higher cellularity, "staghorn" vascularity, and less collagen content) share inversions at the 12q13 locus resulting in a gene fusion between NGFI-A binding protein 2 and signal transducer and activator of transcription 6 (NAB2-STAT6), which can be immunohistochemically detected by aberrant nuclear expression of STAT6 protein $[17,38]$. The tumor may occur at any anatomical site. Within the CNS, a dural-based, supratentorial manifestation is most common, while about $10 \%$ of the cases involve the spinal tract [18]. In the 2016 WHO classification, grade I corresponds to the solitary fibrous tumor phenotype within the histological spectrum and grade II corresponds to the hemangiopericytoma phenotype. Grade III is assigned using a mitotic count of $\geq 5$ mitoses per 10 high-power fields [17]. The behavior in cases with solitary fibrous tumor phenotype is more likely benign, while those with hemangiopericytoma phenotype are at risk of recurrence and metastasis [18].

In 2014, Vandenbussche et al. [8] published a large series of 16 cases with ENM of primary CNS tumors. The majority of cases in their series were diagnosed as hemangiopericytomas $(n=8)$ with meningiomas $(n=3)$ representing the next most frequent tumor entity. One single tumor, an oligodendroglioma, involved the parotid gland. The single glioblastoma in their series was a unique case with manifestation of ENM in a transplant recipient. In contrast to their study, no embryonal tumors such as medulloblastoma were found in our patient population perhaps due to the low frequency of such tumors in adults. Of note, neither institution contributing to our study has a significant pediatric focus. Furthermore, Vandenbussche et al. [8] noted the long latency period prior to ENM for most hemangiopericytomas in their series, which was followed by a somewhat shorter latency period for meningiomas. In our small series, the latency period was highly variable and ranged between few months in the case of a glioblastoma with a primitive neuronal component and almost 2 decades in the case of an atypical meningioma. Although longer patient survival has been proposed as potential factor for the occurrence of ENM, the first case of our series demonstrated clinical manifestation of metastatic disease within an extremely short time period. Hence, the true contribution of survival time remains to be further explored.

Finally, 2 additional potential pitfalls should be kept in mind. One is the extremely rare manifestation of metastatic CNS tumors in serous effusions with [39] or without [40-42] prior shunt placement. The other is the rare occurrence of a substantially different, genuine, or preparation-related tumor morphology in ENM compared to the primary site [13]. No cases with malignant effusion were detected in our patient population, and only the glioblastoma with a primitive neuronal component in our series presented a diagnostic challenge from the perspective that no glial component was detectable in the sample obtained from the metastatic site, which may or may not have been the result of a preferential sampling by FNA. The case, however, also underlines that subtypes or variants of primary CNS tumors can present with ENM and, as such, our case adds to the previously described cytological features of gliosarcoma [43] and small cell glioblastoma [44].

\section{Conclusion}

FNA can be used to accurately diagnose ENM of primary CNS tumors, which, although as rare occurrence, can manifest themselves with extremely variable latency and not infrequently in or around the parotid gland area thus mimicking an inflammatory or neoplastic process unrelated to the primary tumor. Our series demonstrates that the morphological characteristics of the FNA samples show sufficient similarities to the histopathological features of the primary tumors in most cases to establish the relationship between primary and ENM even in variant morphologies. If required, this relationship can be further supported with ancillary studies. The increasing identification and use of molecular parameters to define CNS tumor entities, although not necessary or available 
in any of the cases of our retrospective series, can prove extremely useful in this scenario $[13,32]$ and, consequently, more invasive diagnostic measures can be avoided. The exact mechanism(s) involved in extraneural tumor spread $[45,46]$ and, despite progress made in recent decades [47], more effective treatment options for cases with manifest ENM remain to be identified.

\section{Acknowledgement}

The authors would like to acknowledge Dr. D.G. Munoz (St. Michael's Hospital, Toronto, ON) for providing access to the microscopic slides of the resection specimen for one of the cases in our series and Dr. S.M. Armstrong (University of Toronto, Toronto, $\mathrm{ON}$ ) for support with the translation of one article written in Spanish.

\section{Statement of Ethics}

The study was approved with a waiver of consent by the Research Ethics Boards of the University Health Network (ID\#165026) and the Sunnybrook Health Sciences Centre (ID\#179-2016).

\section{Conflict of Interest Statement}

None of the authors have relevant conflicts of interest.

\section{Funding Sources}

No funding was received for this study.

\section{Author Contributions}

J.S. and Z.G. designed the project. J.S. and L.M. collected the data. L.M. drafted the manuscript. J.S. and Z.G. revised the manuscript critically for important intellectual content. All the authors approved of the final version of the manuscript and agreed to be accountable for all aspects of the work.

\section{Data Availability Statement}

All data generated or analyzed during this study are included in this article. Further enquiries can be directed to the corresponding author.

\section{References}

1 Barnholtz-Sloan JS, Ostrom QT, Cote D. Epidemiology of brain tumors. Neurol Clin. 2018;36(3):395-419.

2 Brenner DR, Weir HK, Demers AA, Ellison LF, Louzado C, Shaw A, et al. Projected estimates of cancer in Canada in 2020. CMAJ. 2020;192(9):E199-205.

3 Lian H, Daniels C, Han YP, Li QF, Zhao Y, Wang BC, et al. Incidence of metastatic disease and survival among patients with newly diagnosed primary CNS tumors in the United States from 2004-2013. J Cancer. 2019;10(13): 3037-45.

4 Amitendu S, Mak SK, Ling JM, Ng WH. A single institution experience of the incidence of extracranial metastasis in glioma. J Clin Neurosci. 2012;19(11):1511-5.

5 Subramanian A, Harris A, Piggott K, Shieff C, Bradford R. Metastasis to and from the central nervous system: the "relatively protected site”. Lancet Oncol. 2002;3(8):498-507.

6 Dolman CL. Lymph node metastasis as first manifestation of glioblastoma. Case report. J Neurosurg. 1974;41(5):607-9.

7 Joggerst KH, Langford LA, Williams MD. Aggressive meningiomas involving the parotid gland. Ann Diagn Pathol. 2012;16(2): $85-90$.

8 Vandenbussche CJ, Ho CY, Nugent SL, Ali SZ. Extraneural metastases of primary central nervous system tumors identified by fine needle aspiration: a retrospective analysis. Acta Cytol. 2014;58(2):117-24.
9 Waite KJ, Wharton SB, Old SE, Burnet NG. Systemic metastases of glioblastoma multiforme. Clin Oncol (R Coll Radiol). 1999; 11(3):205-7.

10 Swinnen J, Gelin G, Fransis S, Vandevenne J, Van Cauter S. Glioblastoma with extracranial parotid, lymph node, and pulmonary metastases: a case report. Radiol Case Rep. 2019; 14(11):1334-47.

11 Taha M, Ahmad A, Wharton S, Jellinek D. Extra-cranial metastasis of glioblastoma multiforme presenting as acute parotitis. $\mathrm{Br} \mathrm{J}$ Neurosurg. 2005;19(4):348-51.

12 Jie W, Bai J, Li B. An extracranial metastasis of glioblastoma mimicking mucoepidermoid carcinoma. World Neurosurg. 2018;116:352-

13 Can B, Akpolat I, Meydan D, Üner A, Kandemir B, Söylemezoğlu F. Fine-needle aspiration cytology of metastatic oligodendroglioma: case report and literature review. Acta Cytol. 2012;56(1):97-103.

14 Liang L, Olar A, Niu N, Jiang Y, Cheng W, Bian XW, et al. Primary glial and neuronal tumors of the ovary or peritoneum: a clinicopathologic study of 11 cases. Am J Surg Pathol. 2016;40(6):847-56.

15 Rushing EJ, Bouffard JP, McCall S, Olsen C, Mena H, Sandberg GD, et al. Primary extracranial meningiomas: an analysis of 146 cases. Head Neck Pathol. 2009;3(2):116-30.

16 Possanzini P, Pipolo C, Romagnoli S, Falleni M, Moneghini L, Braidotti P, et al. Primary extra-cranial meningioma of head and neck: clinical, histopathological and immunohistochemical study of three cases. Acta Otorhinolaryngol Ital. 2012;32(5):336-8.

17 Louis DN, Perry A, Reifenberger G, von Deimling A, Figarella-Branger D, Cavenee WK, et al. The 2016 World Health Organization Classification of Tumors of the Central Nervous System: a summary. Acta Neuropathol. 2016;131(6):803-20.

18 Louis DN, Ohgaki H, Wiestler OD, Cavenee WE, editors. WHO classification of tumours of the central nervous system. Revised 4th ed. Lyon: International Agency for Research on Cancer (IARC); 2016.

19 Perry A, Miller CR, Gujrati M, Scheithauer BW, Zambrano SC, Jost SC, et al. Malignant gliomas with primitive neuroectodermal tumor-like components: a clinicopathologic and genetic study of 53 cases. Brain Pathol. 2009;19(1):81-90.

20 Song X, Andrew Allen R, Terence Dunn S, Fung KM, Farmer P, Gandhi S, et al. Glioblastoma with PNET-like components has a higher frequency of isocitrate dehydrogenase 1 (IDH1) mutation and likely a better prognosis than primary glioblastoma. Int J Clin Exp Pathol. 2011;4(7):651-60.

21 Tamai S, Kinoshita M, Sabit H, Furuta T, Miyashita $\mathrm{K}$, Yoshimura K, et al. Case of metastatic glioblastoma with primitive neuronal component to the lung. Neuropathology. 2019;39(3):218-23. 
22 Ricard JA, Cramer SW, Charles R, Gil Tommee C, Le A, Bell WR, et al. Infratentorial glioblastoma metastasis to bone. World Neurosurg. 2019;131:90-4.

23 Park CC, Hartmann C, Folkerth R, Loeffler JS, Wen PY, Fine HA, et al. Systemic metastasis in glioblastoma may represent the emergence of neoplastic subclones. J Neuropathol Exp Neurol. 2000;59(12):1044-50.

24 Kuhn U, Kohler HH, Jecker P. [Rare tumors of the parotid gland. Lymphadenoma of a sebaceous gland and extracranial metastasis from glioblastoma]. HNO. 2003;51(5):41720.

25 Ogungbo BI, Perry RH, Bozzino J, Mahadeva D. Report of GBM metastasis to the parotid gland. J Neurooncol. 2005;74(3):337-8.

26 Kraft M, Lang F, Braunschweig R, Janzer RC. Parotid gland metastasis from glioblastoma multiforme: a case report and review of the literature. Eur Arch Otorhinolaryngol. 2008; 265(6):709-11.

27 Romero-Rojas AE, Diaz-Perez JA, Amaro D, Lozano-Castillo A, Chinchilla-Olaya SI. Glioblastoma metastasis to parotid gland and neck lymph nodes: fine-needle aspiration cytology with histopathologic correlation. Head Neck Pathol. 2013;7(4):409-15.

28 Taskapilioglu MO, Aktas U, Eser P, Tolunay S, Bekar A. Multiple extracranial metastases from secondary glioblastoma: a case report and review of the literature. Turk Neurosurg. 2013;23(6):824-7.

29 Hamilton JD, Rapp M, Schneiderhan T, Marcel Schneiderhan T, Sabel M, Hayman A, et al. Glioblastoma multiforme metastasis outside the CNS: three case reports and possible mechanisms of escape. J Clin Oncol. 2014; 32(22): $80-4$.

30 Frade Porto N, Delgado Fernández J, García Pallero MLÁ, Penanes Cuesta JR, Pulido Ri- vas P, Gil Simoes R. Subcutaneous tissue metastasis from glioblastoma multiforme: a case report and review of the literature. Neurocirugia. 2019;30(3):149-54.

31 Alhoulaiby S, Abdulrahman A, Alouni G, Mahfoud M, Shihabi Z. Extra-CNS metastasis of glioblastoma multiforme to cervical lymph nodes and parotid gland: a case report. Clin Case Rep. 2020;8(9):1672-7.

32 Wang M, Murphy KM, Kulesza P, Hatanpaa KJ, Olivi A, Tufaro A, et al. Molecular diagnosis of metastasizing oligodendroglioma: a case report. J Mol Diagn. 2004;6(1):52-7.

33 Davis MJ, Hasan F, Weinreb I, Wallace MC, Kiehl TR. Extraventricular anaplastic ependymoma with metastasis to scalp and neck. J Neurooncol. 2011;104(2):599-604.

34 Chao MM, Packer RJ, Myseros JS, Rood BR. Isolated extracranial recurrence of anaplastic ependymoma. Pediatr Blood Cancer. 2011; 56(2):317-8

35 Kim SI, Lee Y, Kim SK, Kang HJ, Park SH. Aggressive supratentorial ependymoma, RELA fusion-positive with extracranial metastasis: a case report. J Pathol Transl Med. 2017;51(6):588-93.

36 Pachella LA, Kamiya-Matsuoka C, Lee EL, Olar A, Yung WK. Supratentorial extraventricular anaplastic ependymoma with extracranial metastasis. J Clin Neurosci. 2015 22(3):605-7.

37 Umbach G, El Ahmadieh TY, Plitt AR, Aoun SG, Neeley OJ, Lyon KA, et al. Extraneural metastatic anaplastic ependymoma: a systematic review and a report of metastases to bilateral parotid glands. Neurooncol Pract. 2020; $7(2): 218-27$.

38 Kristensen BW, Priesterbach-Ackley LP, Petersen JK, Wesseling P. Molecular pathology of tumors of the central nervous system. Ann Oncol. 2019;30(8):1265-78.
39 Stephens S, Tollesson G, Robertson T, Campbell R. Diffuse midline glioma metastasis to the peritoneal cavity via ventriculo-peritoneal shunt: case report and review of literature. J Clin Neurosci. 2019;67:288-93.

40 Lee CC, Jiang JS, Chen ET, Yokoo H, Pan YH, Tsai MD. Cytologic diagnosis of a metastatic oligodendroglioma in a pleural effusion. A case report. Acta Cytol. 2006;50(5):542-4

41 Nauen DW, Li QK. Cytological diagnosis of metastatic glioblastoma in the pleural effusion of a lung transplant patient. Diagn Cytopathol. 2014;42(7):619-23.

42 Hori YS, Fukuhara T, Aoi M, Oda K, Shinno Y. Extracranial glioblastoma diagnosed by examination of pleural effusion using the cell block technique: case report. Neurosurg Focus. 2018;44(6):E8

43 Parwani AV, Berman D, Burger PC, Ali SZ Gliosarcoma: cytopathologic characteristics on fine-needle aspiration (FNA) and intraoperative touch imprint. Diagn Cytopathol. 2004;30(2):77-81.

44 Kalogerak A, Tamiolakis D, Zoi I, KarvelaKalogeraki I, Karvelas-Kalogerakis M, Segredakis J, et al. FNA Cytology in pediatric small cell glioblastoma. Acta Biomed. 2018;89(2): 265-8.

45 Awan M, Liu S, Sahgal A, Das S, Chao ST, Chang EL, et al. Extra-CNS metastasis from glioblastoma: a rare clinical entity. Expert Rev Anticancer Ther. 2015;15(5):545-52.

46 Carvalho JADV, Barbosa CCL, Feher O, Maldaun MVC, Camargo VP, Moraes FY, et al. Systemic dissemination of glioblastoma: literature review. Rev Assoc Med Bras (1992). 2019;65(3):460-8.

47 Cunha MLVD, Maldaun MVC. Metastasis from glioblastoma multiforme: a meta-analysis. Rev Assoc Med Bras (1992). 2019;65(3): 424-33. 